\title{
Family Therapy meets Internal Medicine: Innovations in Medical Family Therapy
}

\author{
Gary H. Bischof ${ }^{1}$ \\ Laura A. Mohr ${ }^{2}$ \\ Monica L. Lieser ${ }^{3}$
}

\begin{abstract}
Medical family therapy has been touted as the next frontier for the field of marriage and family therapy. This article provides an overview of this area of family therapy and identifies some of the key benefits of a collaborative approach to health care. The authors then describe implementation of this approach in an internal medicine practice in Michigan. Finally, a case example illustrates how this type of integrated care is provided.
\end{abstract}

Key Words: Family therapy, collaborative health care, medical family therapy, internal medicine, integrative health care.

${ }^{I}$ Gary H. Bischof, Ph.D. is Associate Professor and Program Director of Marriage and Family Therapy in the Department of Counselor Education and Counseling Psychology at Western Michigan University, 3102 Sangren Hall, Kalamazoo, Michigan, 49008. Electronic mail may be sent via the Internet to gary.bischof@wmich.edu.

${ }^{2}$ Laura A. Mohr, Ph.D. is Director of Family Therapy Connections and Behavioral Medicine at Ingham Regional Medical Center in Lansing, Michigan.

${ }^{3}$ Monica L. Lieser, M.A. is currently employed with Family and Children Services, and is also in private practice with Child and Family Psychological Services, both in Kalamazoo, Michigan.

This article describes a growing area of the field of marriage and family therapy that is termed "medical family therapy" or "collaborative family health care." In short, this area involves collaborative approaches to health care in which health care providers and marriage and family therapists or other family-oriented mental health professionals work together to provide an integrated, biopsychosocial approach to care that actively considers and engages the patient's family or support system in the treatment process.

Michigan Family Review, 9, 1, 19-29, 2004 (C) Michigan Council on Family Relations 


\section{Review of Literature}

Medical family therapy or collaborative family health care has been billed as the "next frontier" of marriage and family therapy (Bloch, 1992). Medical family therapy involves collaboration among family therapists and health care providers who, in more integrated collaborative models, may be practicing in the same facility. This interest in coordinated care has developed in the context of a call for more cost-effective and innovative approaches to health care (McDaniel, Hepworth \& Doherty, 1992; Patterson, Peek, Heinrich, Bischoff, \& Scherger, 2002). Four factors have contributed to the interest in collaborative approaches:

- First, an enormous number of mental health problems are presented in medical settings, particularly in primary care. Up to 40 percent of primary care patients have a diagnosable mental disorder, and one-half to two-thirds of those meeting criteria for a mental disorder go unrecognized (deGruy, 1997).

- Second, a small percentage (10\%) of patients, termed "high utilizers," accounts for 40 to 60 percent of health care expenditures. Half of these high utilizers have psychiatric diagnoses, such as depression or somatization disorder, and frequently have a history of abuse or neglect (Seaburn, Lorenz, Gunn, Gawinski, $\&$ Mauksch, 1996). These high utilizers often are not amenable to a traditional biomedical approach and demand expensive diagnostic procedures that often are unable to detect any observable biomedical cause for their complaints.

- Third, shorter hospital stays and increasing use of outpatient surgery have led to family members taking on greater burdens of health care in the home (McDaniel et al., 1992). Thus, family members are providing significant amounts of informal health care but are infrequently actively involved in the treatment process by medical providers.

- Fourth, advances in health care and prescription medications have meant more focus on chronic illness and lifestyle issues, which often can be greatly influenced by family dynamics and relationships (Campbell, 2003; Rolland, 1994).

Active family involvement can play a significant role in improving treatment adherence over the course of chronic illnesses. In each of these four areas, one can see how the addition of a mental health professional, such as a marriage and family therapist working alongside medical providers, would serve to enhance the overall health care services for patients and their family members.

Given these four factors, family therapists have much to offer health care providers in addressing some of the more difficult and frustrating aspects of their work (Bischof, 1996). For example, issues of noncompliance with medical treatment and

difficulties altering destructive health behaviors such as cigarette smoking or overeating

Michigan Family Review, 9, 1, 19-29, 2004 (C) Michigan Council on Family Relations 
may be understood more clearly as the family system and patient or family beliefs are investigated by the family therapist. Research in the area of compliance or adherence with medical treatment recommendations reveals that only one-third of patients correctly follow physicians' directives, and approximately half of patients are not taking medications as prescribed (Ekstrand \& Chesney, 2002). It has been suggested that noncompliance may be related to the power differential between the physician and patient and that patients may not feel their problems have been adequately listened to or understood or do not fully understand the rationale for the recommended plan of treatment. Although patient non-adherence has generally been viewed as a medical problem, Ekstrand and Chesney suggested that noncompliance is in reality a behavioral issue and that a behavioral treatment plan must be initiated and maintained over time. Others have indicated that patients are more likely to ask questions or express reservations about taking medications to a therapist than to their physician, perhaps because therapists have more time with patients or may be perceived as more collegial (Patterson et al., 2002).

Other areas in which a family therapist can be helpful include: (a) childhood chronic or acute illness and its impact on marital and sibling relationships; (b) problematic relationships between health care providers and patients or families; (c) treatment impasses and triangulation of the patient, family, and physician, or of the patient and competing multiple health care providers; (d) pregnancy loss or infertility; and (e) death or unresolved loss. Medical family therapists can also help to improve the job satisfaction of medical providers by assisting with complicated or challenging patients and by providing general support to over-stressed health care providers (McDaniel et al., 1992; Seaburn et al., 1996). In a qualitative study of the experiences of medical family therapists working in non-academic settings (Bischof, 1999), one participant reported that her joining a rural family medicine practice was a solution devised by a prominent family physician who chaired the local family medicine residency to address the low satisfaction of the solo M.D. in that rural practice. Several years later, the M.D. was still practicing there, and the addition of the medical family therapist seemed to be an effective intervention. In addition to such anecdotal reports, collaborative approaches to health care are supported by a limited but promising number of outcome research studies on this topic.

\section{Efficacy of Family Interventions and Collaborative Family Approaches}

Although outcome studies on collaborative family health care are very limited, there is an established literature on the cost-effectiveness of psychosocial interventions with physical health problems (see Katon \& Gonzalez, 1994; Levenson, 1992; Mumford, Schlesinger, Glass, Patrick, \& Cuerdon, 1984; Mumford \& Schlesinger, 1987 for reviews). Studies show that overall health care expenditures can be offset by the application of psychosocial interventions in many cases, especially when hospital stays are shortened. In most of these studies the treatment providers worked independently, and there was no collaboration or contact between the medical providers and mental health professionals (Campbell, 1996). In a recent review, Campbell (2003) identified four areas 
that have shown sound evidence of the positive impact of family interventions: family caregiving of elders, childhood chronic illness, spouse involvement in chronic adult illnesses, and family involvement in health promotion/disease prevention.

It should be acknowledged that health care providers may feel threatened by patients and family members who are informed and empowered to be actively involved in medical care. For example, work in the area of childhood cancer has shown that staff members may be threatened by the active involvement of parents who are involved in local self-help groups (Chesler, 2002; Lozowski, Chesler, \& Chesney, 1993). Parents who were involved in such support groups intervened in their children's care more frequently, often to prevent or correct a mistake in the medical care. Lozowski et al. called for the "empowerment of parents as active partners in the care of children with cancer and suggested reeducation efforts with staff so that they will appreciate the usefulness of inviting, or responding positively to, parental interventions in care" (p. 63).

In an earlier comprehensive review of the literature on the effectiveness of family interventions in the treatment of physical illness, Campbell and Patterson (1995) concluded the following:

Although a large body of research demonstrates that the family has a powerful influence on physical health, the evidence for the effectiveness of family intervention in physical illness is less conclusive. Family therapy and other family interventions appear to be most effective in chronic childhood illnesses such as asthma and diabetes. Family interventions have also been shown to be effective in the management of some cardiovascular and neurological disorders and for the treatment of obesity. This research supports the increasingly important role of medical family therapy in the new health care system. (p. 545)

There has been very little research conducted on the efficacy of a collaborative approach to health care. In a summary of the literature in this area, only five clinical trials of collaborative care were located (Campbell, 1996). Three of these studies involved very minimal collaboration, a single psychiatric consultation. Collectively, the results of these studies are encouraging, but far from conclusive. Two well-designed trials by Katon and colleagues (Katon et al., 1992, 1995) suggest that interventions involving greater contact and collaboration between primary care physicians and mental health professionals are more likely to result in improved outcomes. Campbell (p. 137) concluded that the field of collaborative family health care "desperately needs more studies examining the process and outcome of different approaches to collaborative care." The fact that some large managed care organizations, typically driven by keeping costs at a minimum, are implementing collaborative approaches to care supports the utility of this approach (Fischer, Heinrich, Davis, Peek, \& Lucas, 1997). But before there is widespread acceptance, more quality studies need to be done to demonstrate that collaborative care results in improved health outcomes and is cost-effective.

Michigan Family Review, 9, 1, 19-29, 2004 (C) Michigan Council on Family Relations 
Indeed, one of the recent emphases in the collaborative family health care field includes conducting sound outcome research on collaborative approaches. Other current emphases are on refining and operationalizing models of collaborative care, expanding collaboration with medical specialties and subspecialties beyond family medicine, and on influencing decision makers in health care policy and managed care (Patterson et al., 2002; Seaburn et al., 1996). This article focuses on two of these trends: the relatively recent expansion of medical family therapy to the primary care medical specialty of internal medicine and the implementation of a viable model of a collaborative familyoriented clinic in a hospital-based internal medicine practice in Michigan. The next section describes the collaborative clinic, followed by a case example that illustrates how this type of collaborative arrangement is implemented and can benefit both practitioners and families.

\section{Internal Medicine Collaborative Healthcare Center}

The Collaborative Healthcare Center (CHC), affiliated with Ingham Regional Medical Center in Lansing, Michigan, consists of three health care practices: Michigan State Adult Medicine, an internal medicine physician group practice; Okemos Internal Medicine, an internal medicine residency clinic; and Family Therapy Connections, a marriage and family therapy clinic. All three clinics serve individuals and families in an effort to maximize health and well-being. Physicians, therapists, nurses, receptionists, medical assistants, therapy interns and medical residents are all dedicated to offering collaborative care and services to individuals in the mid-Michigan area.

\section{Internal Medicine_-Prime for Collaborative Care}

Internal medicine is a primary care specialty that treats adults $(18$ years +$)$ and excludes obstetrics. Patients cared for in an internal medicine clinic tend to be older and often have more complicated and/or chronic illnesses than other general medical practice settings. The presence of multiple illnesses presents physicians with patients in need of additional services beyond biomedical attention (e.g., loss of ability, adjustment to new diagnosis). The aging population also faces unique developmental struggles, such as grief over the loss of loved ones, and end of life issues that are seen more frequently in an internal medicine practice. These many issues present tremendous opportunity for mental health providers to offer essential services to a population much in need of support.

\section{Getting Started}

The CHC grew out of initial contacts made by the second author, a marriage and family therapist interested in working with patients seen by internal medicine physicians. The therapist sought out physicians and offered a way to support them in their struggle with "difficult" patients, those who many physicians refer to as "thick-chart" patients or "high utilizers." In addition, the physicians quickly recognized the appropriateness of referrals for patients diagnosed with such common ailments as depression and anxiety. 
Physicians responded favorably as mental health services were introduced as a way to help physicians, to "make their lives easier."

In order to obtain new referrals and to build a practice, it was necessary for the therapists to continue offering services to support physicians and to integrate professionally into the medical culture. When physicians refer patients to another provider or specialist, it is standard operating procedure for the outside provider to report the findings and recommended treatment back to the referring physician. In the collaborative setting, mental health providers should operate in a similar manner. Establishing this process is necessary to integrate mental health processes into this unique medical environment. The case example below details how the therapist responds to the referral and establishes a collaborative relationship with the referring physician from the outset.

As professional, collaborative relationships develop, opportunities for further education of physicians arise. The need for referrals to mental health providers for assistance with depression and anxiety or for help with "difficult" patients is relatively apparent to physicians. In addition, when patients express extreme emotions during their medical visits or discuss frustrations with their personal lives, physicians recognize the importance of referrals to mental health providers. Relevant opportunities also exist, however, when health care issues are presented to physicians (e.g., increased blood pressure, new diagnosis of diabetes, migraine headaches) that are not as obviously affected by psychosocial components. These present ongoing opportunities to educate physicians regarding the role of mental health on physical well being.

Essential components of developing the $\mathrm{CHC}$ collaborative practice have been:

- To address existing needs of physicians and to support them with patients with whom they struggle (i.e., 'thick chart patients')

- To be sensitive to the medical culture

- To continue informal discussions with physicians, looking for opportunities to provide education regarding the potential effect of psychosocial influences on healthcare

- To enhance the collaborative nature of healthcare by including patient participation in treatment.

\section{Ongoing Collaboration}

Today, the $\mathrm{CHC}$ includes private practices for internal medicine physicians as well as for a marriage and family therapist. In addition, the $\mathrm{CHC}$ is a teaching clinic. Trainees include internal medicine residents and marriage and family therapy (MFT) student interns. The training environment offers several advantages. Resident physicians 
tend to be more receptive to new ideas and therefore are interested in collaboration. Residents are also new and welcome support in the management of patients. Finally, MFT interns have the opportunity to learn about the culture of medicine while developing therapy skills and the ability to work within that culture.

The private practitioners at the $\mathrm{CHC}$ have been collaborating around patient care for several years. Once service providers develop the paradigm to see patients in their entirety (physical and socioemotional), it is difficult to treat patients otherwise. Teaching faculty, physicians, and the marriage and family therapist exemplify a collaborative paradigm through everyday interactions with patients, medical residents, and therapy interns. Education is both formal, in the form of lectures, as well as informal case-based, in the form of ongoing collaborative patient care. Residents and interns are encouraged to develop working relationships with each other and develop skills for collaboration around patient care. The goal of the center is to offer an education to interns and residents that allows for optimal patient care in an integrative, biopsychosocial manner that is effective and consistent with best practices in the current literature.

\section{A Case Illustration}

The following case description illustrates an example of the kinds of cases that may be seen in such a collaborative health care setting and details the nature of the collaboration between the physician and therapist involved in this patient's care. The case involves ongoing collaboration between an internal medicine third year resident physician and an MFT intern. The interactions described below took place over a onemonth period of time, including the consultation by the therapist intern in the medical exam room prior to the establishment of formal therapy sessions. The patient's name and identifying information have been altered to protect confidentiality.

\section{Pre-therapy/In-room Consultation}

During a scheduled doctor's appointment regarding severe swelling of the eyelids, Savannah K., approximately 40 years old, presented as tearful and overwhelmed. This was Savannah's first visit to the clinic. She had recently moved to the area and needed to establish ongoing health care. Along with attending to her physical symptoms, the resident physician was also sensitive to Savannah's emotional distress. He verbally acknowledged her tearfulness and then invited one of the MFT interns into the room for a brief consultation. Prior to this in-room consultation, Savannah had no previous encounter with a mental health clinician.

During the consultation Savannah reported having been diagnosed four years ago with Lupus. Lupus Erythematosus (Lupus) is a chronic autoimmune disease that involves the body producing antibodies that attack its own bodily tissue. Symptoms may include a butterfly-shaped rash on the face, headache, fatigue, arthritic joint disease, heart damage, shortness of breath, and impaired kidney function. The disease is irregular, with remissions and flare-ups that may often be incapacitating to the patient. The cause of 
Lupus remains unknown, leading to limited clarity about diagnosis and treatment (Encarta, 2004; Gill, Ouisel, Rocca, \& Walters, 2003).

Savannah explained her frustrations related to all of the "unknowns" surrounding the disease and its systemic nature. Savannah also reported having "roller-coaster" like moods pertaining to her feelings surrounding the illness, ranging from anger and sadness to feelings of empowerment and perseverance. The MFT intern normalized and empathized with Savannah's range of emotions and invited her to weekly therapy sessions where she could begin to attend to and sort out her feelings. Savannah accepted the invitation and called for an appointment later the same week.

\section{Therapy}

As of this writing, Savannah had attended three consecutive, 50-minute weekly therapy sessions. In these sessions Savannah initially set goals for therapy. Her goals were two-fold: (a) she desired a place to talk freely about her illness, and (b) she also wanted to learn ways of building relationships outside of therapy, in which she could also feel safe to talk freely about the illness. Although the therapist met individually with Savannah, a family systems orientation was embraced by the therapist. One of the client's primary goals was related to social and family relationships, especially regarding her ability to talk openly with others about her health concerns and gain support from others. Interestingly, family therapists working in medical settings may see clients individually more often than a family therapist in a traditional mental health practice (Bischof, 1999). This may be due in part to a focus on the individual patient in conventional medical culture in which patients are typically seen and referred as individuals by their physicians.

Therapy sessions included Savannah telling her diagnosis "story" and welcoming feelings that came up for her in reflections; exploring her perspective on the illness and how this empowers her to fight the battle of dealing with this illness; and, along those same lines, looking at the illness as separate from her as a person-viewing the illness as outside of her. This last notion of speaking about the illness as outside the person is similar to the technique of externalizing from narrative approaches to therapy (White \& Epston, 1990) and has been identified as a useful and empowering way to talk with patients about their illness (McDaniel et al., 1992).

During one of Savannah's sessions she participated in an art therapy intervention, during which she drew the changes in her body and talked about what she actively does to attend to these changes. Savannah revealed that she finds the element of water healing and comforting. Knowing that Lupus often brings discomfort to the muscular/skeletal system, the MFT intern wondered if water aerobics would aid Savannah physically, emotionally, and socially. When the MFT intern shared this thought with Savannah, she was noticeably moved by the idea and showed genuine enthusiasm to participate, pending her physician's support.

Michigan Family Review, 9, 1, 19-29, 2004 (C) Michigan Council on Family Relations 


\section{Collaboration}

As noted above, the initial contact about this patient between the referring physician and MFT intern occurred when the physician called the therapist into the exam room after the patient became emotionally upset during a regular medical visit. This availability and immediate accessibility of an on-site therapist enhances the opportunity for timely collaboration and permits the physician to obtain support on complicated cases or for acute mental health concerns. The relationship that a patient has built with his or her physician can also be more easily transferred through the brief face-to-face interaction that on-site collaboration affords. The therapist also responds to the patient in a time of need and makes a personal connection that, of course, would not be possible if the therapist were not practicing in the same setting. Further, it is believed that this onsite availability makes follow up with a mental health referral more likely and facilitates an earlier start of mental health treatment (Seaburn et al., 1996).

During the first therapy session, the MFT intern reviewed with Savannah the guidelines for therapy surrounding confidentiality and clarified the nature of the communication that the therapist would likely have with Savannah's physician, with her consent. Savannah reported finding this collaborative arrangement comforting, due to the amount of attention her illness required from her physician and that she clearly saw her emotional distress as connected to her illness.

Collaboration continued when Savannah's physician was informally contacted following Savannah's initial formal intake session. The MFT intern approached the physician during a brief passing in the office; such "curbside" consultations are a common way communication occurs in the busy culture of medicine (McDaniel et al., 1992; Seaburn et al., 1996). They discussed briefly the physiological nature of Lupus and the appropriateness of Savannah's range of emotions as related to the illness. A formal contact was made following the second session with Savannah. A letter was sent to the physician thanking him for his sensitive referral and outlining the structure of therapy and the goals mutually set by Savannah and the therapist. This letter was placed in Savannah's medical chart. After Savannah's third therapy session, the MFT intern again informally approached the resident physician in regards to water aerobic therapy. The physician was highly receptive and supported this activity for both its physical and emotional benefits.

In the future, based on one of Savannah's goals for therapy, the opportunity may present itself for Savannah to ask someone to accompany her to water aerobics class. This would provide the potential space to build a safe relationship where Savannah can speak freely about her feelings relating to the Lupus, while surrounding herself with the physical and emotional comfort of water. Savannah might also be referred to a Lupus support group, should one exist in her area. Consistent with her goals and the transition of moving to a new community, Savannah will also be encouraged to develop social relationships that do not necessarily relate to her illness in an effort to establish adequate social support and allow for time and attention away from the Lupus. As therapy 
progresses, the MFT intern may also have Savannah consider inviting significant members of her social support system to therapy sessions, if appropriate. The therapist will also continue to collaborate with the referring physician to coordinate care.

\section{Conclusion}

This article described the growing area of medical family therapy, a specialized focus in the field of marriage and family therapy. We selected the case example of a woman with Lupus to illustrate the communication and collaboration between the mental health therapist and physician around an illness that lends itself well to a biopsychosocial perspective. Future developments in this burgeoning area of family therapy will likely include continued integration of family therapy with other medical specialties, stronger outcome research about the effectiveness and cost-effectiveness of such approaches, and the development of models that may provide more holistic and integrative alternatives to traditional western biomedicine.

\section{References}

Bischof, G. H. (1996). Family systems medicine. In F. Piercy, D. Sprenkle, J. Wetchler, \& Associates, Family therapy sourcebook ( $2^{\text {nd }}$ ed., pp. 338-349). New York: Guilford.

Bischof, G. H. (1999). Medical family therapists working in non-academic medical settings: A phenomenological study (Doctoral dissertation, Purdue University, 1999). Dissertation Abstracts International, 60, 5428.

Bloch, D. (1992, March). Family systems medicine: The next frontier. Presentation at the Family Therapy Networker Symposium, Washington, DC.

Campbell, T. L. (1996). Research reports: Clinical trials of collaborative healthcare. Families, Systems \& Health, 14, 137-144.

Campbell, T. L. (2003). The effectiveness of family interventions for physical disorders. Journal of Marital and Family Therapy, 29, 263-281.

Campbell, T. L., \& Patterson, J. M. (1995). The effectiveness of family interventions in the treatment of physical illness. Journal of Marital and Family Therapy, 21, 545-583.

Chesler, M. (2002). Support organizations for parents of children with cancer: Local, national, and international problems and prospects. In F. M. Cancian \& D. Kurz (Eds.), Child care and inequality: Rethinking carework for children and youth (pp. 174-192). New York: Routledge.

deGruy, F. V. (1997). Mental health care in the primary care setting: A paradigm problem. Families, Systems \& Health, 15, 3-26.

Encarta (2004). Lupus Erythematosus. Retrieved February 17, 2004, from http://www.encarta.msn.com/encyclopedia_761561727/Lupus.html 
Ekstrand, M. L., \& Chesney, M. A. (2002). Adhering to complex medication regimens. In M. A. Chesney \& M. H. Antoni (Eds.), Innovative approaches to health psychology: Prevention and treatment lessons from AIDS (pp. 283-306). Washington, DC: USical Association.

Fischer, J. L., Heinrich. R., Davis, T., Peek, C., \& Lucas, S. (1997). Mental health and primary care in an HMO. Families, Systems \& Health, 15, 379-391.

Gill, J., Ouisel, A., Rocca, P., \& Walters, D. (2003). Diagnosis of systemic Lupus Erythematosus. American Family Physician, 68, 2179-2186.

Katon, W. \& Gonzalez, J. (1994). A review of randomized trials of psychiatric consultationliaison studies in primary care. Psychosomatics, 35, 268-278.

Katon, W., Von Korff, M., Lin, E., Bush, T., Russo, J., Lipscomb, P., \& Wagner, E. (1992). A randomized trial of psychiatric consultation with distressed high utilizers. General Hospital Psychiatry, 14, 86-98.

Katon, W., Von Korff, M., Lin, E., Walker, E., Simon, G., Bush, T., Robinson, P., \& Russo, J. (1995). Collaborative management to achieve treatment guidelines: Impact on depression in primary care. Journal of the American Medical Association, 273, 1026-1031.

Levenson, J. L. (1992). Psychosocial interventions in chronic medical illness: An overview of outcome research. General Hospital Psychiatry, 14S, 43S-49S.

Lozowski, S., Chesler, M. A., \& Chesney, B. K. (1993). Parental intervention in the medical care of children with cancer. Journal of Psychosocial Oncology, 11 (3), 63-88.

McDaniel, S., Hepworth, J., \& Doherty, W. (1992). Medical family therapy: A biopsychosocial approach to families with health problems. New York: Basic Books.

Mumford, E. \& Schlesinger, H. J. (1987). Assessing consumer benefit: Cost offset as an incidental effect of psychotherapy. General Hospital Psychiatry, 9, 360-363.

Mumford, E., Schlesinger, H. J., Glass, G., Patrick, C. \& Cuerdon, T. (1984). A new look at evidence about reduced cost of medical utilization following mental health treatment. American Journal of Psychiatry, 141, 1145-1158.

Patterson, J., Peek, C. J., Heinrich, R., Bischoff, R., \& Scherger, J. (2002). Mental health professionals in medical settings: A primer. New York: Norton.

Rolland, J. (1994). Families, illness, \& disability: An integrative treatment model. New York: Basic Books.

Seaburn, D., Lorenz, A., Gunn, W., Gawinski, B., \& Mauksch, L. (1996). Models of collaboration: A guide for mental health professionals working with health care practitioners. New York: Basic Books.

White, M., \& Epston, D. (1990). Narrative means to therapeutic ends. New York: Norton. 